ARTICLE

Received 21 Mar 2016 | Accepted 22 Apr 2016 | Published 23 May 2016

DOl: $10.1038 /$ ncomms11711

OPEN

\title{
Spin-valley locking in the normal state of a transition-metal dichalcogenide superconductor
}

L. Bawden1, S.P. Cooil², F. Mazzola2, J.M. Riley¹,3, L.J. Collins-Mclntyre1, V. Sunko1,4, K. W. B. Hunvik², M. Leandersson ${ }^{5}$, C.M. Polley ${ }^{5}$, T. Balasubramanian' ${ }^{5}$, T.K. Kim³ ${ }^{3}$ M. Hoesch ${ }^{3}$, J.W. Wells ${ }^{2}$, G. Balakrishnan ${ }^{6}$, M.S. Bahramy ${ }^{7,8}$ \& P.D.C. King ${ }^{1}$

Metallic transition-metal dichalcogenides (TMDCs) are benchmark systems for studying and controlling intertwined electronic orders in solids, with superconductivity developing from a charge-density wave state. The interplay between such phases is thought to play a critical role in the unconventional superconductivity of cuprates, Fe-based and heavy-fermion systems, yet even for the more moderately-correlated TMDCs, their nature and origins have proved controversial. Here, we study a prototypical example, $2 \mathrm{H}-\mathrm{NbSe}_{2}$, by spin- and angle-resolved photoemission and first-principles theory. We find that the normal state, from which its hallmark collective phases emerge, is characterized by quasiparticles whose spin is locked to their valley pseudospin. This results from a combination of strong spin-orbit interactions and local inversion symmetry breaking, while interlayer coupling further drives a rich three-dimensional momentum dependence of the underlying Fermi-surface spin texture. These findings necessitate a re-investigation of the nature of charge order and superconducting pairing in $\mathrm{NbSe}_{2}$ and related TMDCs.

\footnotetext{
${ }^{1}$ SUPA, School of Physics and Astronomy, University of St Andrews, St Andrews, Fife KY16 9SS, UK. ${ }^{2}$ Department of Physics, Norwegian University of Science and Technology (NTNU), N-7491 Trondheim, Norway. ${ }^{3}$ Diamond Light Source, Harwell Campus, Didcot, OX11 ODE, UK. ${ }^{4}$ Max Planck Institute for Chemical Physics of Solids, Nöthnitzer Straße 40, 01217 Dresden, Germany. ${ }^{5}$ MAX IV Laboratory, Lund University, PO Box 118, 22100 Lund, Sweden. ${ }^{6}$ Department of Physics, University of Warwick, Coventry CV4 7AL, UK. ${ }^{7}$ Quantum-Phase Electronics Center and Department of Applied Physics, The University of Tokyo, Tokyo 113-8656, Japan. ${ }^{8}$ RIKEN center for Emergent Matter Science (CEMS), Wako 351-0198, Japan. Correspondence and requests for materials should be addressed to P.D.C.K. (email: philip.king@st-andrews.ac.uk).
} 
$\mathrm{n}$ combination with broken structural inversion symmetry, spin-orbit coupling (SOC) provides a powerful route to stabilize spin-polarized electronic states without magnetism. This can give rise to electrically-tuneable spin splittings via the Rashba effect, promising new technological developments in spintronics ${ }^{1}$, and underpins the formation of spin-helical Dirac cones at the surfaces of topological insulators ${ }^{2}$. It is strongly desired to realize similar effects in systems where more pronounced electronic interactions drive the emergence of collective phases. In non-centrosymmetric superconductors, for example, spin-splitting driven by strong SOC is expected to induce a mixing of spin-triplet and singlet superconducting order parameters $^{3}$, and offers potential for stabilizing topological superconductors ${ }^{4}$. Yet, identifying suitable candidate materials has proved a major challenge to date. Partly this is driven by a relative dearth of non-centrosymmetric metals, while for non-magnetic systems in which the centre of inversion is maintained, robust-spin degeneracies of their electronic states would typically be expected due to the dual constraints of time-reversal and inversion symmetry.

In contrast, we show here from spin- and angle-resolved photoemission (ARPES) measurements that the normal state of the centrosymmetric TMDC superconductor $2 \mathrm{H}-\mathrm{NbSe}_{2}$ (hereafter denoted $\mathrm{NbSe}_{2}$ ) hosts a strong layer-resolved and momentum-dependent spin polarization of its electronic states at and in the vicinity of the Fermi level. We attribute this as a consequence of a recently-realized form of spin polarization that can emerge in globally centrosymmetric materials in which constituent structural units nonetheless break inversion symmetry ${ }^{5-7}$. Together with first-principles calculations, we show how this drives a critical and complex interplay of interlayer interactions and $\mathrm{SOC}$ in $\mathrm{NbSe}_{2}$. This yields a rich underlying spin-polarized electronic landscape from which charge order and superconductivity emerge upon cooling.

\section{Results}

Bulk electronic properties of $\mathrm{NbSe}_{2}$. We consider here exclusively the layered $2 \mathrm{H}$ polymorph (Fig. 1a). Each layer forms a graphene-like honeycomb structure with $\mathrm{Nb}$ occupying the $\mathrm{A}$ sublattice and two Se atoms situated on the B sublattice. These lie out of the basel plane, equidistant above and below the transition metal. The unit cell contains two such layers, stacked along the $c$-axis with $180^{\circ}$ in-plane rotation. Our resistivity measurements (Fig. 1b) from single-crystal $\mathrm{NbSe}_{2}$ samples show a metallic temperature-dependence. They additionally exhibit a pronounced hump at a temperature of $T_{\mathrm{CDW}} \approx 33 \mathrm{~K}$ indicative of charge-density wave (CDW) formation ${ }^{8}$, as well as a sharp superconducting transition at $T_{\mathrm{c}} \approx 7 \mathrm{~K}$. The corresponding normal-state Fermi surface is shown in Fig. 1c, as measured by ARPES. There are two barrels centred around each zone-corner $\overline{\mathrm{K}}\left(\overline{\mathrm{K}}^{\prime}\right)$ point, which are strongly trigonally-warped. Two further barrels are centred at the $\bar{\Gamma}$ point, the inner of which is hexagonal, while the outer exhibits additional warping. From our first-principles density-functional theory (DFT) calculations, and consistent with previous studies ${ }^{9-11}$, we assign all four of these Fermi surface sheets as being predominantly derived from $\mathrm{Nb} 4 d$ orbitals. Additional spectral weight at the zone centre is evident in our ARPES measurements for selected photon energies, which we attribute as a fifth, highly three-dimensional, Fermi surface sheet of predominantly Se $p_{z}$ orbital character.

This sheet also contributes diffuse filled-in intensity, due to the finite out-of-plane momentum $\left(k_{z}\right)$ resolution of ARPES, close to the zone centre in measurements of the electronic dispersions along in-plane high-symmetry directions (Fig. 2a). The $\mathrm{Nb}$-dominated states, on the other hand, yield clear spectral features close to their Fermi crossings. Kinks in their measured dispersion and a decrease in linewidth near the Fermi-level point to relatively strong electron-phonon coupling in this system ${ }^{11-13}$. It is these $\mathrm{Nb}$-derived states that are known to host the largest energy gaps at the Fermi level arising from the CDW and superconducting instabilities in this system ${ }^{13-16}$, although the origins of these have proved highly controversial ${ }^{8,10,11,13-22}$. To the best of our knowledge, all prior theoretical treatments assume these orders emerge from an electronic liquid of trivially spin-degenerate character. Indeed, standard expectations of group theory would say this must be the case for the centrosymmetric space group $\left(\mathrm{P}_{3} / \mathrm{mmc}\right)$ of $2 \mathrm{H}-\mathrm{NbSe}_{2}$.

In contrast, we directly observe pronounced spin polarizations of the underlying electronic states in spin-resolved energy distribution curves (spin-EDCs, see Methods) measured along the $\bar{\Gamma}-\overline{\mathrm{K}}$ direction, shown in Fig. $2 \mathrm{c}-\mathrm{f}$. Close to the saddle point of these bands (Fig. 2d), two clearly-separated peaks can be observed in spin-EDCs. The measured spin polarization is almost entirely out-of-plane (Fig. 2d and Supplementary Fig. 1), with a sign that reverses between the two bands. We attribute this as a

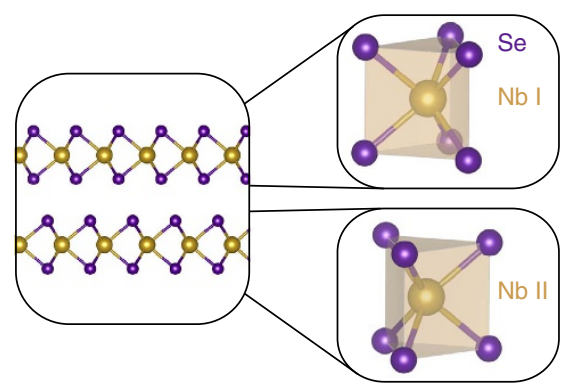

b

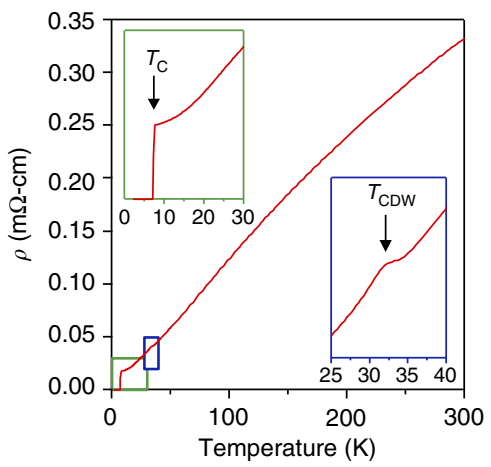

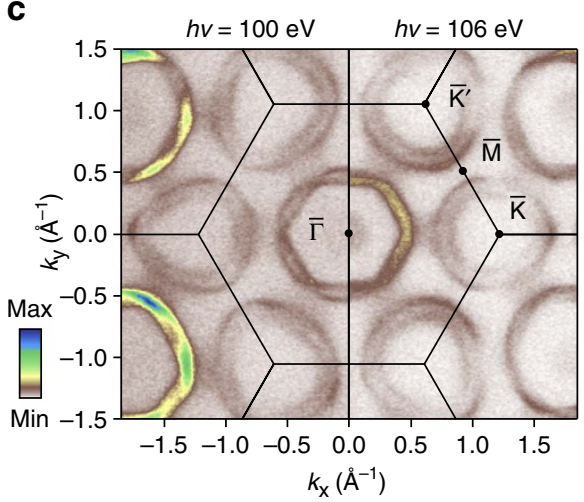

Figure 1 | Superconductivity and charge-density wave order in $\mathbf{2} \mathbf{H}-\mathbf{N b S e}_{\mathbf{2}}$. (a) Centrosymmetric bulk crystal structure (side view) of $2 \mathrm{H}-\mathrm{NbSe} \mathrm{e}_{2}$. This is formed by stacking non-centrosymmetric layers of $D_{3 h}$ symmetry with $180^{\circ}$ relative rotations, restoring the bulk inversion centre. (b) Resistivity measurements show clear signatures of charge-density wave formation at $T_{\mathrm{CDW}} \approx 33 \mathrm{~K}$ and superconductivity at $T_{\mathrm{c}} \approx 7 \mathrm{~K}$ (magnified in the insets). (c) Normal-state Fermi surface measured by ARPES with $h v=100 \mathrm{eV}$ (left-hand-side) and $h v=106 \mathrm{eV}$ (right-hand-side); $E_{F} \pm 20$ meV. This consists of two $\mathrm{Nb}$-derived barrels centred around the zone-corner $\overline{\mathrm{K}}\left(\overline{\mathrm{K}}^{\prime}\right)$ points, two Nb-derived barrels at the Brillouin zone centre and an additional central diffuse pocket (most visible at $h v=106 \mathrm{eV}$ ) predominantly derived from Se $p_{z}$ orbitals. 
a

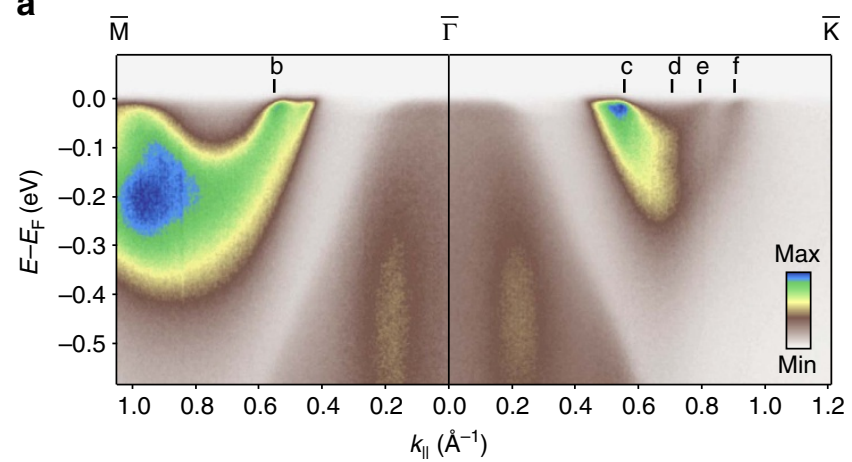

b

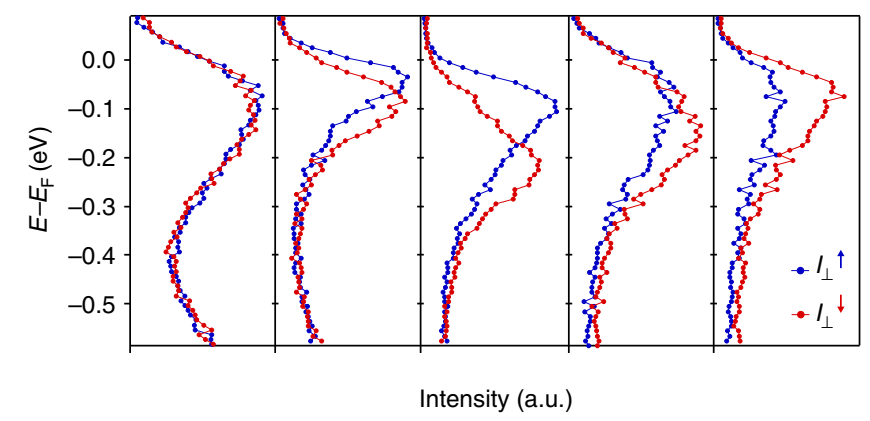

Figure 2 | Spin-polarized bulk electronic structure. (a) Dispersion measured by ARPES ( $h v=22 \mathrm{eV}$ ) along the $\bar{M}-\bar{\Gamma}-\bar{K}$ direction. (b-f) Spin-resolved EDCs at the momenta marked in $\mathbf{a}$, revealing a strong spin polarization of these electronic states along $\bar{\Gamma}-\bar{K}$. The horizontal axis tick marks denote the zero of each EDC.

arising from local inversion symmetry breaking within the individual layers that make up the bulk crystal structure ${ }^{5,6,23}$. For sufficiently weak interlayer interactions (a point we return to below), pronounced SOC characteristic of the $4 d$ transition metal can lift the spin degeneracy of the states localized within each layer, that thus strongly feel the local inversion asymmetry. A layer-dependent sign change of this spin polarization, mediated by the rotated-layer stacking of the bulk crystal structure, would act to restore overall spin degeneracy as required by global inversion and time-reversal symmetries. Nonetheless, the electronic states retain strong layer-resolved spin polarizations. Depth-averaging probes would largely be insensitive to these, but photoemission is a surface sensitive technique. Incoherent superposition of photoelectrons emitted from neighbouring layers of the unit cell would lead to some suppression of the measured spin polarization, while interference effects can further complicate this picture ${ }^{5}$. Nonetheless, the extreme surface sensitivity at the photon energies used here, with an inelastic mean free path on the order of the interlayer separation, renders us predominantly sensitive to the spin texture of the top-most layer of the unit cell.

Spin-valley locking. Our measurements show how such spin textures in $\mathrm{NbSe}_{2}$ persist up to the Fermi level. This is evident in spin-resolved EDCs along the $\bar{\Gamma}-\overline{\mathrm{K}}$ direction (Fig. 2c-f) as well as the spin polarization of EDCs and a Fermi-level momentum-distribution curve along the $\overline{\mathrm{K}}-\overline{\mathrm{M}}-\overline{\mathrm{K}}^{\prime}$ direction (Fig. 3). The latter clearly reveals how the spin polarization reverses sign for each pair of Fermi surface sheets centred on neighbouring $\mathrm{K}$ and $\mathrm{K}^{\prime}$ points. This is a natural consequence of time-reversal symmetry. Here, this results in a coupling of the spin to the so-called valley index, the quantum number which distinguishes $\overline{\mathrm{K}}$ - and $\overline{\mathrm{K}}^{\prime}$-centred Fermi surfaces in $\mathrm{NbSe}_{2}$ (Fig. 3e). Such spin-valley coupling has recently been extensively investigated for the band extrema in monolayers of semiconducting TMDCs ${ }^{24-26}$, where it has not only been shown to lead to new physics, such as a valley Hall effect ${ }^{27}$, but also to offer potential for devices exploiting the valley pseudospin ${ }^{28,29}$. Our observations here point to a pronounced role of spin-valley coupling also for the low-energy quasiparticle excitations of the metallic $2 \mathrm{H}$-structured TMDCs.

Critically, it is from this spin-polarized Fermi sea that electron-hole and electron-electron pairing interactions drive the formation of CDW order and superconductivity. The largest $\mathrm{CDW}$ gaps in $\mathrm{NbSe}_{2}$ are located on the zone-corner spin-valleylocked Fermi surfaces ${ }^{13}$. The CDW wave vector is entirely in-plane ${ }^{30}$, making the hidden layer-dependent spin polarizations relevant. The underlying spin textures can also be expected to have important implications for superconductivity. Recent measurements on electrically gated $\mathrm{MoS}_{2}$, which is known to host a strong spin-valley locking in its band structure ${ }^{25,26}$, have found evidence for unconventional so-called Ising superconductivity ${ }^{31,32}$. In this, the pronounced spin-orbit field that induces the underlying spin texture of the electronic states pins the spin of Cooper pair electrons in the out-of-plane direction. This leads to upper critical fields dramatically exceeding the Pauli paramagnetic limit for a magnetic field applied within the $a b$-plane. Similar phenomenology has recently been reported for mono- and few-layer $\mathrm{NbSe}_{2}$ (ref. 33), entirely consistent with our direct observation of spin-valley locking in this compound.

In bulk $\mathrm{NbSe}_{2}$, the spin-layer locking, and also multi-band nature as compared to gated $\mathrm{MoS}_{2}$, raises further prospects for stabilizing a delicate balance between different pairing states. In the normal state, we find that the $\bar{\Gamma}$-centred Fermi surface barrels, which are known to support a modulated superconducting gap in the bulk ${ }^{13,34}$, are also strongly spin-polarized along the $\bar{\Gamma}-\overline{\mathrm{K}}$ direction (Fig. 2c). Their spin polarization, however, is completely suppressed along $\bar{\Gamma}-\bar{M}$ (Fig. 2b). Intriguingly, the largest superconducting gaps of this Fermi surface determined in Rahn et al. $^{13}$ are located at the regions of strongest spin polarization evident here. Pronounced superconducting gaps are also known to occur on the zone-corner Fermi surfaces ${ }^{13,15,34}$, which as demonstrated above, host strong spin-valley locking even in the bulk. Given the pronounced influence of SOC, a mixing of spin-triplet and spin-singlet order parameters could potentially be expected $^{3}$. Forming even pseudospin-singlet Cooper pairs from the strongly spin-polarized Fermi surfaces could necessitate a phase locking between the order parameter of neighbouring Fermi surface sheets. Moreover, the $c$-axis coherence length in $\mathrm{NbSe}_{2}$ is much greater than the interlayer separation ${ }^{35,36}$. This raises the tantalizing prospect that the inherent coupling between the spin and layer pseudospins reported here could be tuned to drive an instability to an odd-parity pair density-wave state, where the sign of the superconducting gap becomes tied to the layer index 7,37 . The proximity of bulk $\mathrm{NbSe}_{2}$ and similar compounds to such phases requires further theoretical exploration, and will depend sensitively on the relative importance of inter- and intra-band as well as interlayer pairing interactions.

Three-dimensional spin structure. Our DFT calculations (Fig. 4) already reveal a key role of interlayer interactions in mediating and controlling the underlying spin texture of the normal-state 
a

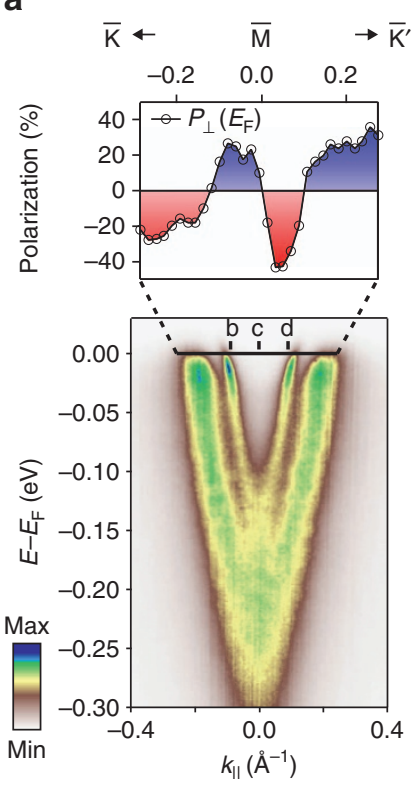

b

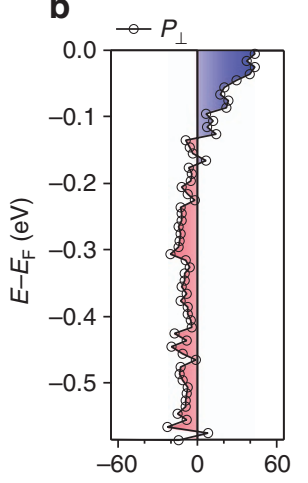

c

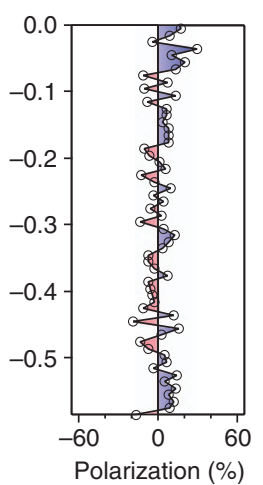

d

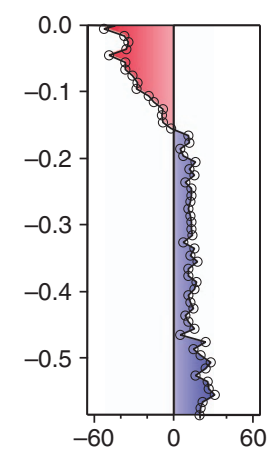

e

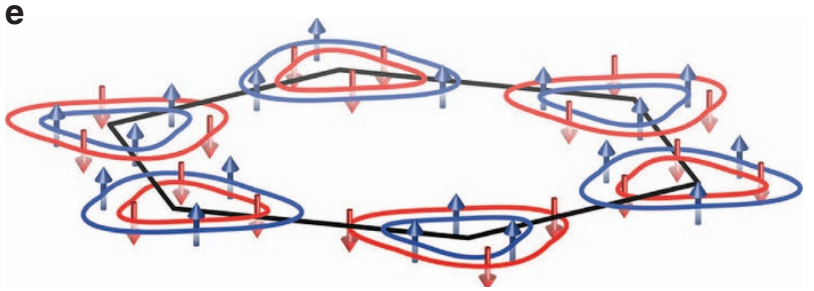

Figure 3 | Spin-valley locked Fermi surfaces. (a) Dispersion measured ( $h v=22 \mathrm{eV}$ ) along the $\bar{K}-\bar{M}-\bar{K}^{\prime}$ direction, together with the corresponding spin polarisation of a momentum distribution curve at the Fermi level and (b-d) EDCs at the momenta marked in $\mathbf{a}$. These reveal how the sign of the spin polarization for each zone-corner Fermi surface sheet becomes locked to the valley degree of freedom, as shown schematically in $\mathbf{e}$.

a

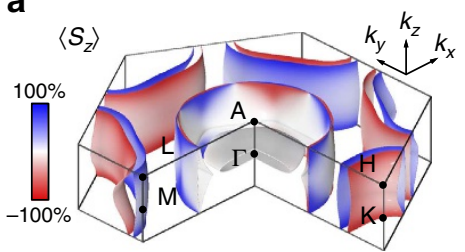

b

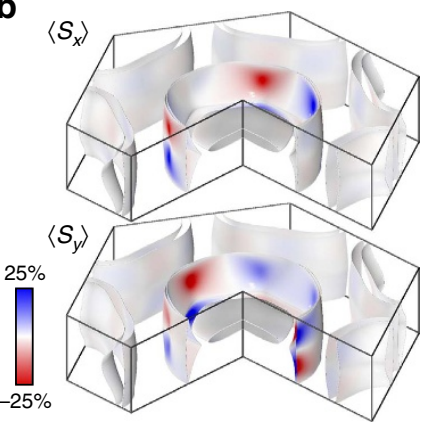

C

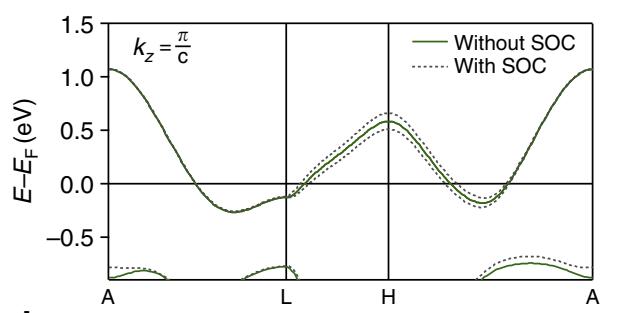

d

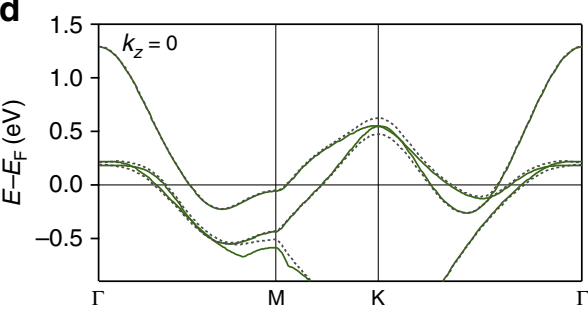

e

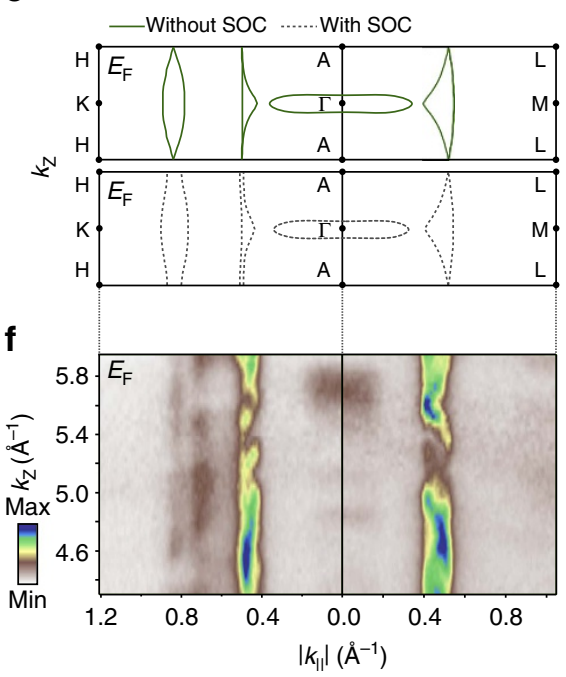

Figure 4 | Interplay of interlayer interactions and intralayer inversion symmetry breaking. (a,b) DFT calculations of the (a) out-of-plane and (b) in-plane spin polarization of the three-dimensional Fermi surface of $\mathrm{NbSe}_{2}$ projected onto the first layer of the unit cell. (c, d) Calculated electronic structure along (c) A-L-H-A $\left(k_{z}=\pi / C\right)$ and (d) $\Gamma-\mathrm{M}-\mathrm{K}-\Gamma\left(k_{z}=0\right)$ with and without SOC. (e) Corresponding influence of SOC on the Fermi surface contours in the $\Gamma-\mathrm{K}-\mathrm{H}-\mathrm{A}$ and $\Gamma-\mathrm{M}-\mathrm{L}-\mathrm{A}$ planes (shown throughout the full three-dimensional Brillouin zone in Supplementary Fig. 2). (f) Our experimental ARPES measurements of such $k_{z}$-dependent Fermi surfaces $(h v=60-130 \mathrm{eV})$ are in good general agreement with the theoretical calculations including SOC, supporting a SOC-mediated suppression of interlayer hopping in the $\Gamma-\mathrm{K}-\mathrm{H}-\mathrm{A}$ plane.

bulk Fermi surface. The calculated spin polarization projected onto the first layer of the unit cell is shown throughout the full three-dimensional Brillouin zone in Fig. 4a,b. The momentumdependent spin polarizations are determined by the effective spin-orbit field, $\mathbf{B}_{\mathbf{s o}}=\beta(\nabla \mathbf{V} \times \mathbf{k})$, where $\beta$ is a momentumdependent scaling factor, $\nabla \mathbf{V}$ is the net electrostatic potential gradient and $\mathbf{k}$ is the crystal momentum. The horizontal mirror symmetry of each $\mathrm{NbSe}_{2}$ structural unit about the transitionmetal plane ( $\sigma_{\mathrm{h}}$ of the $\mathrm{D}_{3 h}$ point group; Fig. 1a) ensures that $\nabla \mathbf{V}$ is entirely within the $x y$ plane. Due to the $180^{\circ}$ relative rotation of neighbouring $\mathrm{NbSe}_{2}$ monolayers in the bulk crystal structure, $\nabla \mathbf{V}$, and thus $\mathbf{B}_{\mathbf{s o}}$, has opposite sign for successive layers in the unit cell. This causes the sign of the spin polarization to reverse at each momentum-space point when projected onto layer 2 versus layer 1 of the unit cell (Supplementary Fig. 3). This confirms that local inversion asymmetry within each $\mathrm{NbSe}_{2}$ layer drives the formation of the spin-polarized states observed here. 
For electronic states whose wavefunctions are completely delocalized over both layers of the unit cell, the spin-orbit field from each layer cancels. Such states are consequently unpolarized, restoring the conventional expectations for a centrosymmetric space group. This can be observed for the highly threedimensional pancake-like Fermi surface at the Brillouin zone centre here. In contrast, for electronic states predominantly localized within individual layers of the unit cell, strong layerdependent spin-orbit fields mediate large layer-resolved spin polarizations. This is ideally realized at the three-dimensional Brillouin zone boundary along $k_{z}\left(k_{z}=\pi / c\right)$. In a tight-binding picture, for this $k_{z}$, interlayer hoppings within a unit cell and between neighbouring unit cells are out of phase with each other and thus cancel ${ }^{10}$. This can be directly visualized by comparing the dispersion of the electronic states along the $\Gamma-\mathrm{M}-\mathrm{K}-\Gamma$ and A-L-H-A directions: neglecting SOC, a single fourfold degenerate band crosses the Fermi level in the $k_{z}=\pi / c$ plane (Fig. 4c), whereas this is split into a pair of twofold degenerate bands by interlayer interactions for $\left|k_{z}\right|<\pi / c$, as evident in Fig. $4 \mathrm{~d}$.

With its forbidden interlayer coupling, the electronic structure for the $k_{z}=\pi / c$ plane is thus formally equivalent to that of an isolated monolayer. The spin-orbit field is consequently maximized, driving the largest (>90\%) layer-dependent spin-polarizations. These are purely out-of-plane (Fig. 4a,b), as $(\nabla \mathbf{V} \times \mathbf{k})$ must lie entirely along $z$ at the Brillouin zone boundary along $k_{z}$. Along the A-L direction, however, a vertical mirror plane in the crystal structure forbids any out-of-plane component of $(\nabla \mathbf{V} \times \mathbf{k})$. The spin-orbit field must therefore be strictly zero along A-L. This enforces a touching, and hence spin degeneracy, of the zone-centre Fermi surface barrels along this direction, which are otherwise strongly spin-polarized throughout the $k_{z}=\pi / c$ plane.

For other $k_{z}$ through the Brillouin zone, the spin-orbit field strength can be partially suppressed by finite interlayer coupling. Moreover, the $z$-component of the momentum induces a non-zero component of $(\nabla \mathbf{V} \times \mathbf{k})$ in the $x y$ plane. Together, this causes not only the magnitude of the Fermi surface spin polarization, but also its vectorial spin texture, to develop a strong dependence on both the in- and out-of-plane momentum (Fig. 4a,b). Within the $\Gamma$-M-L-A plane, the calculated Fermi surface crossings are relatively strongly dispersive in $k_{z}$ (Fig. 4e). This is consistent with our experimental measurements of the $k_{z}$-dependent Fermi surface (Fig. 4f) and points to significant interlayer coupling. Even away from $k_{z}=\pi / c$, where there are strict spin degeneracies along $\mathrm{A}-\mathrm{L}$ as discussed above, the Fermi surface spin polarizations therefore become strongly suppressed in the entire vicinity of the $\Gamma-\mathrm{M}$-L-A plane.

For the $\Gamma-\mathrm{K}-\mathrm{H}-\mathrm{A}$ plane, on the other hand, we find that the $k_{z}$ dispersion of the Fermi surface crossings are significantly reduced by the inclusion of spin-orbit coupling in the calculations (Fig. 4e), in keeping with our experimental measurements of more two-dimensional Fermi contours for this plane (Fig. 4f). This reduction in $k_{z}$ dispersion is achieved by a lifting, via SOC, of the fourfold degeneracy that would otherwise be present along the $\mathrm{A}-\mathrm{H}$ line, and is accompanied by the emergence of strong spin polarization of the Fermi surface crossings for $k_{z}=\pi / c$. This is in contrast to the A-L line, where the energetic degeneracy of the Fermi crossings is protected by their symmetry-enforced spin degeneracy. The reduction of $k_{z}$ dispersion within the $\Gamma-\mathrm{K}-\mathrm{H}-\mathrm{A}$ plane is equivalent to a spin-orbit-mediated suppression of interlayer hopping here ${ }^{28}$. This allows relatively strong spin polarizations to be maintained in the vicinity of this plane, with only a moderate suppression of the spin-orbit field strength away from $k_{z}=\pi / c$.

For momenta close to the K-H line, the in-plane momentum is always much larger than the out-of-plane component. This causes $(\nabla \mathbf{V} \times \mathbf{k})$ to remain predominately aligned along $z$. The spin polarizations of the zone-corner Fermi surfaces are thus largely out-of-plane throughout the full Brillouin zone (Fig. 4a). For the $\mathrm{Nb}$-derived zone-centre Fermi surface barrels, however, much stronger (up to $\sim 25 \%$ ) in-plane components develop (Fig. $4 \mathrm{~b}$ ). The in-plane spin texture is largely radial to the Fermi surface (Supplementary Fig. 4), and switches sign about the $k_{z}=0$ plane. This indicates a non-zero component of $(\nabla \mathbf{V} \times \mathbf{k})$ within the plane, with a direction that is tied to the sign of $k_{z}$. It thus confirms that an in-plane component of the spin texture arises due to finite out-of-plane momentum, where no symmetry constraint exists to cancel the in-plane component of the spin-orbit field for low-symmetry momenta with $0<\left|k_{z}\right|<\pi / c$. The existence of such hidden in-plane spin textures is a unique property of the bulk compound. Indeed, the in-plane spin component goes strictly to zero at the Brillouin zone boundary along $k_{z}$, enforced by symmetry, and recovering a monolayer-like purely out-of-plane spin texture for $k_{z}= \pm \pi / c$. The bulk system thus hosts a rich intertwining of spin, orbital and layer degrees of freedom, mediating a three-dimensional nature of its spin texture that can be expected to further modulate its pairing interactions ${ }^{31}$.

\section{Discussion}

Taken together, our results show how the combination of interlayer hopping and intralayer inversion symmetry breaking can lead to particularly rich momentum-dependent spin textures of metallic TMDCs. Suppression of Fermi-surface spin polarization away from the Brillouin zone boundary along $k_{z}$ will be reduced with decreased interlayer interactions. Indeed, upper critical fields, already known to exceed the Pauli limit for bulk $\mathrm{NbSe}_{2}$ (ref. 38), are dramatically enhanced in other $2 \mathrm{H}$ TMDC superconductors as a function of increasing interlayer separation $^{39}$. This points to a susceptibility of the bulk systems to Ising superconductivity similar to that recently observed in isolated monolayers ${ }^{33}$. The delicate balance between interlayer hopping and $\mathrm{SOC}$ strength in $\mathrm{NbSe}_{2}$ makes this an ideal material for understanding, and ultimately controlling the role of layer-dependent spin polarizations on the collective states and phases of transition-metal dichalcogenides.

\section{Methods}

ARPES. Spin-resolved ARPES measurements were performed at the I3 beamline of MAX IV Laboratory, Sweden, and ARPES measurements were performed using the I05 beamline of Diamond Light Source, UK. Measurements were performed at temperatures of $50-80 \mathrm{~K}$ using $p$-polarized synchrotron light. Scienta R4000 hemispherical electron analyzers were utilized for all measurements. This was additionally fitted with a mini-Mott detector scheme for the spin-ARPES measurements, configured to simultaneously probe the out-of-plane and in-plane (along the analyzer slit direction) component of the photoelectron $\operatorname{spin}^{40}$. The finite spin-detection efficiency was corrected using a Sherman function of $S=0.17$ (ref. 40), and the spin-resolved EDCs determined according to

$$
I_{i}^{\uparrow, \downarrow}=I_{i}^{\mathrm{tot}}\left(1 \pm P_{i}\right) / 2
$$

with $i=\{\perp, \|\}, I_{i}^{\text {tot }}=\left(I_{i}^{+}-I_{i}^{-}\right), I_{i}^{ \pm}$the measured intensity on the individual detectors in the Mott scattering chamber, corrected by a relative detector efficiency calibration, and the total spin polarization,

$$
P_{i}=\frac{\left(I_{i}^{+}-I_{i}^{-}\right)}{S\left(I_{i}^{+}+I_{i}^{-}\right)} .
$$

Calculations. DFT calculations including SOC were performed using the modified Becke-Johnson exchange potential and Perdew-Burke-Ernzerhof correlation functional implemented in the WIEN2K programme ${ }^{41}$. A $20 \times 20 \times 10 k$-mesh was employed. The DFT results were downfolded using maximally localized Wannier functions ${ }^{42-44}$, employing $\mathrm{Nb} 4 d$ orbitals and Se $5 p$ orbitals as basis functions. The resulting tight-binding Hamiltonian allows a direct extraction of the spin and layer projections of the electronic structure. 
Data availability. Data underpinning this publication can be accessed at http://dx.doi.org/10.17630/edd53357-41e3-4313-9e5e-a0e7780ef4be.

\section{References}

1. Manchon, A., Koo, H. C., Nitta, J., Frolov, S. M. \& Duine, R. A. New perspectives for Rashba spin-orbit coupling. Nat. Mater. 14, 871-882 (2015).

2. Hasan, M. Z. \& Kane, C. L. Colloquium: topological insulators. Rev. Mod. Phys. 85, 3045 (2010).

3. Gor'kov, L. P. \& Rashba, E. I. Superconducting 2D system with lifted spin degeneracy: mixed singlet-triplet state. Phys. Rev. Lett. 87, 037004 (2001).

4. Sato, M. \& Fujimoto, S. Topological phases of noncentrosymmetric superconductors: edge states, Majorana fermions, and non-Abelian statistics. Phys. Rev. B 79, 094504 (2009).

5. Riley, J. M. et al. Direct observation of spin-polarized bulk bands in an inversion-symmetric semiconductor. Nat. Phys 10, 835-839 (2014).

6. Zhang, X., Liu, Q., Luo, J.-W., Freeman, A. J. \& Zunger, A. Hidden spin polarization in inversion-symmetric bulk crystals. Nat. Phys. 10, 387-393 (2014).

7. Sigrist, M. et al. Superconductors with staggered non-centrosymmetricity. J. Phys. Soc. Jpn. 83, 061014 (2014).

8. Wilson, J. A., Di Salvo, F. J. \& Mahajan, S. Charge-density waves in metallic, layered, transition-metal dichalcogenides. Phys. Rev. Lett. 32, 882 (1974).

9. Rossnagel, K. et al. Fermi surface of $2 \mathrm{H}-\mathrm{NbSe}_{2}$ and its implications on the charge-density-wave mechanism. Phys. Rev. B 64, 235119 (2001).

10. Johannes, M. D., Mazin, I. I. \& Howells, C. A. Fermi-surface nesting and the origin of the charge-density wave in $2 \mathrm{H}-\mathrm{NbSe}_{2}$. Phys. Rev. B 73, 205102 (2006).

11. Flicker, F. \& van Wezel, J. Charge order from orbital-dependent coupling evidenced by $\mathrm{NbSe}_{2}$. Nat. Commun. 6, 7034 (2015).

12. Weber, F. et al. Electron-phonon coupling and the soft phonon mode in $\mathrm{TiSe}_{2}$. Phys. Rev. Lett. 107, 107403 (2011).

13. Rahn, D. J. et al. Gaps and kinks in the electronic structure of the superconductor $2 \mathrm{H}-\mathrm{NbSe}_{2}$ from angle-resolved photoemission at $1 \mathrm{~K}$. Phys. Rev. B 85, 224532 (2012).

14. Kiss, T. et al. Charge-order-maximized momentum-dependent superconductivity. Nat. Phys. 3, 720-725 (2007).

15. Yokoya, T. et al. Fermi surface sheet-dependent superconductivity in 2H-NbSe ${ }_{2}$. Science 294, 2518-2520 (2001).

16. Borisenko, S. V. et al. Two energy gaps and Fermi-surface 'arcs' in $\mathrm{NbSe}_{2}$. Phys. Rev. Lett. 102, 166402 (2009).

17. Xi, X. et al. Strongly enhanced charge-density-wave order in monolayer $\mathrm{NbSe}_{2}$. Nat. Nano 10, 765-769 (2015).

18. Rice, T. M. \& Scott, G. K. New mechanism for a charge-density-wave instability. Phys. Rev. Lett. 35, 120 (1975).

19. Shen, D. W. et al. Primary role of the barely occupied states in the charge density wave formation of $\mathrm{NbSe}_{2}$. Phys. Rev. Lett. 101, 226406 (2008).

20. Rossnagel, K. On the origin of charge-density waves in select layered transition-metal dichalcogenides. J. Phys. Cond. Matter. 23, 21 (2011)

21. Soumyanarayanan, A. et al. Quantum phase transition from triangular to stripe charge order in $\mathrm{NbSe}_{2}$. P. Natl Acad. Sci. USA 110, 1623-1627 (2013).

22. Laverock, J. et al. $k$-resolved susceptibility function of $2 \mathrm{H}$-TaSe $\mathrm{T}_{2}$ from angle-resolved photoemission. Phys. Rev. B 88, 035108 (2013).

23. Riley, J. M. et al. Negative electronic compressibility and tunable spin splitting in $\mathrm{WSe}_{2}$. Nat. Nano 10, 1043-1047 (2015).

24. Xiao, D., Liu, G.-B., Feng, W., Xu, X. \& Yao, W. Coupled spin and valley physics in monolayers of $\mathrm{MoS}_{2}$ and other group-VI dichalcogenides. Phys. Rev. Lett. 108, 196802 (2012).

25. Mak, K. F., He, K., Shan, J. \& Heinz, T. F. Control of valley polarization in monolayer $\mathrm{MoS}_{2}$ by optical helicity. Nat. Nano 7, 494-498 (2012).

26. Zeng, H., Dai, J., Yao, W., Xiao, D. \& Cui, X. Valley polarization in $\mathrm{MoS}_{2}$ monolayers by optical pumping. Nat. Nano 7, 490-493 (2012).

27. Mak, K. F., McGill, K. L., Park, J. \& McEuen, P. L. The valley Hall effect in $\mathrm{MoS}_{2}$ transistors. Science 344, 1489-1492 (2014).

28. Gong, Z. et al. Magnetoelectric effects and valley-controlled spin quantum gates in transition metal dichalcogenide bilayers. Nat. Commun. 4, 2053 (2013).

29. Xu, X., Yao, W., Xiao, D. \& Heinz, T. F. Spin and pseudospins in layered transition metal dichalcogenides. Nat. Phys. 10, 343-350 (2014).

30. Moncton, D. E. et al. Neutron scattering study of the charge-density wave transitions in $2 \mathrm{H}-\mathrm{TaSe}_{2}$ and $2 \mathrm{H}-\mathrm{NbSe}_{2}$. Phys. Rev. B 16, 801 (1977).

31. Saito, Y. et al. Superconductivity protected by spin-valley locking in gate-tuned $\mathrm{MoS}_{2}$. Nat. Phys. 12, 144-149 (2016).

32. Lu, J. M. et al. Evidence for two-dimensional Ising superconductivity in gated $\mathrm{MoS}_{2}$. Science 350, 1353-1357 (2015).
33. Xi, X. et al. Ising pairing in superconducting $\mathrm{NbSe}_{2}$ atomic layers. Nat. Phys. 12, 139-143 (2016).

34. Huang, C. L. et al. Experimental evidence for a two-gap structure of superconducting $\mathrm{NbSe}_{2}$ : a specific-heat study in external magnetic fields. Phys. Rev. B 76, 212504 (2007).

35. Sanchez, D., Junod, A., Muller, J., Berger, H. \& Lévy, F. Specific heat of $2 \mathrm{H}-\mathrm{NbSe}_{2}$ in high magnetic fields. Phys. B 204, 167-175 (1995).

36. Nader, A. \& Monceau, P. Critical field of $2 \mathrm{H}-\mathrm{NbSe}_{2}$ down to $50 \mathrm{mK}$. SpringerPlus 3, 16 (2014).

37. Yoshida, T., Sigrist, M. \& Yanase, Y. Pair-density wave states through spinorbit coupling in multilayer superconductors. Phys. Rev. B 86, 134514 (2012)

38. Foner, S. \& McNiff, Jr E. J. Upper critical fields of layered superconducting $\mathrm{NbSe}_{2}$ at low temperature. Phys. Lett. A 45, 429-430 (1973).

39. Klemm, R. A. Pristine and intercalated transition metal dichalcogenide superconductors. Phys. C 514, 86-94 (2015).

40. Berntsen, M. H. et al. A spin- and angle-resolving photoelectron spectrometer. Rev. Sci. Instrum. 81, 035104 (2010).

41. Blaha, P. et al. WIEN2K package, version 10.1. Available at http://www.wien2k.at (2010).

42. Souza, I. et al. Maximally localized Wannier functions for entangled energy bands. Phys. Rev. B 65, 035109 (2001).

43. Mostofi, A. A. et al. Wannier90: a tool for obtaining maximally localised Wannier functions. Comp. Phys. Commun. 178, 685699 (2008).

44. Kuneš, J. et al. WIEN2WANNIER: from linearized augmented plane waves to maximally localized Wannier functions. Comp. Phys. Commun. 181, 1888-1895 (2010).

\section{Acknowledgements}

We thank M.R. Lees for assistance with the transport measurements and C. Hooley, P. Wahl, A.P. Mackenzie, S. Lee and B. Braunecker for useful discussions. We gratefully acknowledge support from the Engineering and Physical Sciences Research Council, UK (work at St Andrews under Grant No. EP/I031014/1 and work at Warwick under Grant No. EP/M028771/1) and the International Max Planck partnership. P.D.C.K. acknowledges support from the Royal Society through a University Research Fellowship. M.S.B. was supported by the Grant-in-Aid for Scientific Research (S) (No. 24224009) from the Ministry of Education, Culture, Sports, Science and Technology (MEXT) of Japan. L.B., J.M.R. and V.S. acknowledge studentship funding from EPSRC through Grant Nos. EP/G03673X/1, EP/L505079/1 and EP/L015110/1, respectively. The experiments at MAX IV Laboratory were made possible through funding from the Swedish Research Council and the Knut and Alice Wallenberg Foundation. We also thank Diamond Light Source for access to beamline I05 (Proposal No. SI11383) that contributed to the results presented here.

\section{Author contributions}

The experimental data were measured by L.B., S.P.C., F.M., J.M.R., L.J.C-M., V.S., K.W.B.H., C.M.P., J.W.W. and P.D.C.K., and analyzed by L.B.; theoretical calculations were performed by M.S.B; G.B. synthesized the samples used in this study; M.L., C.M.P. T.B., T.K.K. and M.H. maintained the synchrotron ARPES end stations and provided experimental support; P.D.C.K., M.S.B. and L.B. wrote the manuscript, with input and discussions from all co-authors; P.D.C.K. conceived the study and was responsible for overall project planning and direction.

\section{Additional information}

Supplementary Information accompanies this paper at http://www.nature.com/ naturecommunications

Competing financial interests: The authors declare no competing financial interests.

Reprints and permission information is available online at http://npg.nature.com/ reprintsandpermissions/

How to cite this article: Bawden, L. et al. Spin-valley locking in the normal state of a transition-metal dichalcogenide superconductor. Nat. Commun. 7:11711 doi: 10.1038/ncomms11711 (2016).

This work is licensed under a Creative Commons Attribution 4.0 International License. The images or other third party material in this article are included in the article's Creative Commons license, unless indicated otherwise in the credit line; if the material is not included under the Creative Commons license, users will need to obtain permission from the license holder to reproduce the material. To view a copy of this license, visit http://creativecommons.org/licenses/by/4.0/ 